\title{
Brachyury proteins regulate target genes through modular binding sites in a cooperative fashion
}

\author{
Thomas Kusch, ${ }^{1,3}$ Tobias Storck, ${ }^{2}$ Uwe Walldorf, ${ }^{2,4}$ and Rolf Reuter ${ }^{1,5,6}$ \\ ${ }^{1}$ Institut für Genetik, Universität zu Köln, 50931 Köln, Germany; ${ }^{2}$ Institut für Genetik, Universität Hohenheim, \\ 70593 Stuttgart, Germany
}

Brachyury proteins, a conserved subgroup of the $T$ domain transcription factors, specify gut and posterior mesoderm derivatives throughout the animal kingdom. The $T$ domain confers DNA-binding properties to Brachyury proteins, but little is known how these proteins regulate their target genes. We characterized a direct target gene of the Drosophila Brachyury-homolog Brachyenteron. Brachyenteron activates the homeobox gene orthopedia in a dose-dependent manner via multiple binding sites with the consensus $(\mathrm{A} / \mathrm{G})(\mathrm{A} / \mathrm{T})(\mathrm{A} / \mathrm{T}) \mathrm{NTN}(\mathrm{A} / \mathrm{G}) \mathrm{CAC}(\mathrm{C} / \mathrm{T}) \mathrm{T}$. The sites and their A/T-rich flanking regions are conserved between D. melanogaster and Drosophila virilis. Reporter assays and site-directed mutagenesis demonstrate that Brachyenteron binding sites confer in part additive, in part synergistic effects on otp transcription levels. This suggests an interaction of Brachyenteron proteins on the DNA, which we could map to a conserved motif within the $\mathrm{T}$ domain. Mouse Brachyury also interacts with Brachyenteron through this motif. We further show that the Xenopus and mouse Brachyury homologs activate orthopedia expression when expressed in Drosophila embryonic cells. We propose that the mechanisms to achieve target gene expression through variable binding sites and through defined protein-protein interactions might be conserved for Brachyury relatives.

[Key Words: Brachyenteron; Brachyury; orthopedia; T domain; protein-DNA interaction]

Received July 23, 2001; revised version accepted December 15, 2001.

T domain factors regulate developmental processes such as oogenesis, gastrulation, neurogenesis, and limb development throughout the animal kingdom (for review, see Smith 1999) and form a super family of at least seven subgroups (Wattler et al. 1998). The best-characterized subgroup are the Brachyury-type $\mathrm{T}$ domain proteins that have been found in ascidians, cephalochordates, sea urchins, star fish, hemichordates, jellyfish, and insects (Smith 1999; Technau and Bode 1999; Shoguchi et al. 2000). The Brachyury homologs are expressed in the gut and the posterior mesoderm where they appear to have virtually the same functions in the different phyla (Kusch and Reuter 1999; Smith 1999; Bassham and Postlethwait 2000; Woollard and Hodgkin 2000). In some clades, the expression in either of the tissues is transient or missing; in these cases the respective aspect of

Present addresses: ${ }^{3}$ Howard Hughes Medical Institute, Department of Biochemistry and Molecular Biology, 306 Althouse Laboratory, The Pennsylvania State University, University Park, PA 16802, USA; ${ }^{4}$ Universität des Saarlandes, FR 2.1 Anatomie und Zellbiologie, Abt. Entwicklungsbiologie, 66421 Homburg/Saar, Germany; ${ }^{5}$ Interfakultäres Institut für Zellbiologie, Universität Tübingen, 72076 Tübingen, Germany. ${ }^{6}$ Corresponding author.

E-MAIL rolf.reuter@uni-tuebingen.de; FAX 49-7071-295-128.

Article and publication are at http://www.genesdev.org/cgi/doi/10.1101/ $\operatorname{gad} .213002$.
Brachyury function might have been lost during evolution (e.g., Peterson et al. 1999; Bassham and Postlethwait 2000).

Brachyury proteins act as transcriptional activators and can bind to a 24-bp palindromic target sequence, which was identified based on the binding site selection method (Kispert and Herrmann 1993). X-ray crystallography revealed an association of the target sequence with two T domains, which are in close contact on the palindrome (Müller and Herrmann 1997). However, the arrangement of binding sites in a palindrome is not a requirement for transcriptional activation by Brachyury: certain combinations of 12-bp halves of the palindromic sequence are sufficient (Kispert et al. 1995). Likewise, the binding sites so far characterized in vivo were solely homologous to palindrome halves. Within the regulatory region of $e f g f$, the first identified target gene of the Xenopus Brachyury protein $\mathrm{Xbra}$, only two half sites were found (Casey et al. 1998).

The Drosophila Brachyury homolog Brachyenteron (Byn) acts in the development of the hindgut and of certain muscle precursors of the midgut (Kispert et al. 1994; Singer et al. 1996; Kusch and Reuter 1999). Byn certainly functions through a larger set of target genes in both developmental processes. One of those is the homeobox 
gene orthopedia (otp; Simeone et al. 1994), which is regulated by Byn specifically in the hindgut (Singer et al. 1996). Here, we show that otp is a direct target of Byn and use $o t p$ as a model to study the transcriptional regulation of a gene by a Brachyury-type transcription factor. The cis-regulatory region of $o t p$ contains multiple binding sites of two types, apparently of high (type A) and of low affinity (type B) to Byn, respectively. Mutations in these sites do not entirely abolish specific binding by Byn, but reduce their affinity and significantly affect transcriptional activation by Byn. Luciferase reporter assays in embryos as well as in cell culture show that type $\mathrm{B}$ binding sites are insufficient for luciferase activation, but stimulate transcription when combined with type A sites in an antiparallel orientation. Thus, Byn binds cooperatively to DNA leading to synergistic effects on target gene activation. The complete set of 15 Byn sites within the regulatory region of otp seems to ensure the high level of otp expression. A conserved region of the T domain is sufficient for a physical interaction between Byn molecules and between Byn and mouse Brachyury proteins in a yeast two-hybrid assay. Moreover, the type A Byn sites show a high homology to the synthetic Brachyury half-site (Kispert and Herrmann 1993), and the mouse Brachyury protein can activate otp expression in the Drosophila embryo. The molecular mechanisms found here for Drosophila Brachyenteron appear to be a highly conserved feature of $\mathrm{T}$ domain transcription factors.

\section{Results}

Multiple upstream regulatory elements regulate gut-specific expression of orthopedia

Based on its expression pattern, the homeobox gene otp was a likely candidate for a direct target of Byn. otp mRNA expression becomes detectable shortly after the onset of byn expression in the common primordium of hindgut and anal pads (in the following only referred to as the hindgut primordium). byn is necessary for the gutspecific otp expression (Singer et al. 1996) and can activate ectopic otp expression when overexpressed by means of the GAL4/UAS system (Fig. 1F,G). We therefore characterized the otp locus and found two transcription start sites, one for hindgut-specific transcripts, another $\sim 5.8 \mathrm{~kb}$ further upstream for CNS-specific transcripts (Fig. 1C). During later stages of embryogenesis otp becomes expressed in the CNS, an aspect independent of byn (Singer et al. 1996).

To identify the hindgut-specific regulatory elements within the otp locus, we fused various genomic fragments of the locus to lac $Z$ reporters and monitored the transgenic embryos for $\beta$-galactosidase expression in the hindgut. A $1.8-\mathrm{kb}$ XhoI/EcoRI fragment $\sim 950$ bp upstream of the putative hindgut-specific transcription start site confers lac $Z$ expression in the hindgut primordium (Fig. 1A,B) in a pattern identical to endogenous otp. lacZ is strongly expressed throughout the hindgut including anal pads, at lower levels at the distal part of the
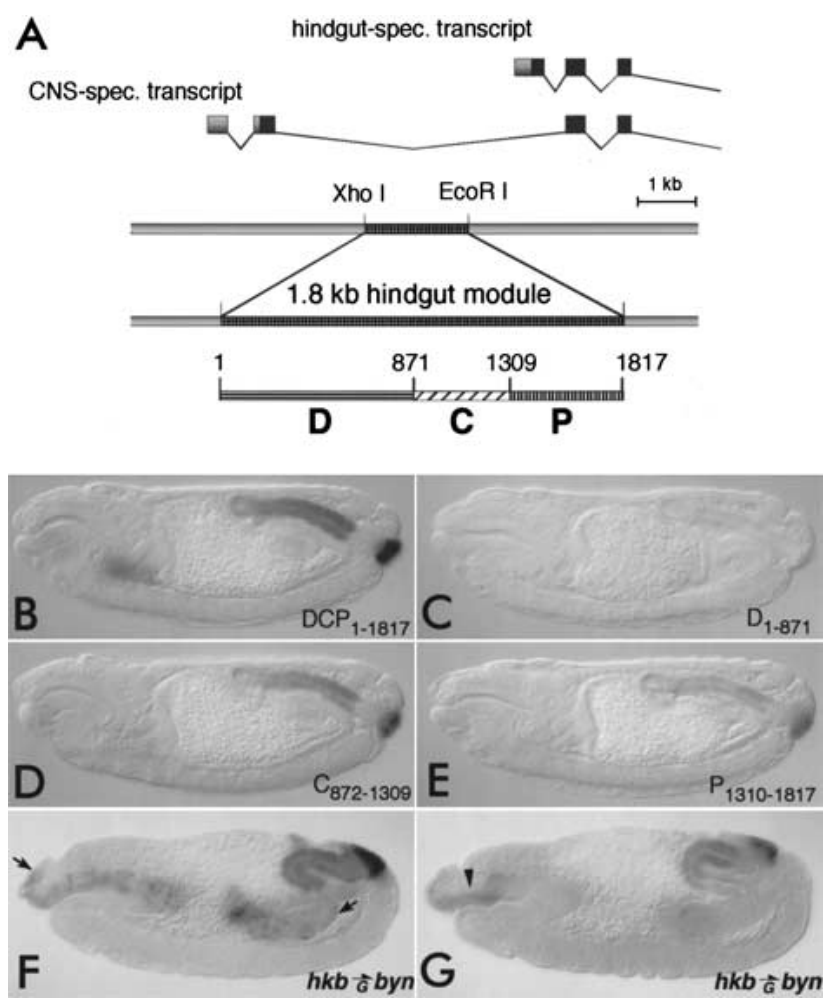

Figure 1. Byn is sufficient to activate hindgut-specific otp expression via distinct regulatory elements. $(A)$ The promoter regions of the otp locus. The CNS-specific and the hindgut-specific otp transcripts are expressed from two different promoters. A 1.8-kb XhoI/EcoRI fragment is sufficient for hindgut-specific otp expression. It was divided into three fragments $\left(\mathrm{D}_{1-871}\right.$, $\left.\mathrm{C}_{872-1309}, \mathrm{P}_{1310-1817}\right)$ using Sau3A. (B-E) Immunodetection of Escherichia coli $\beta$-galactosidase in embryos carrying lacZ reporters (stage 15). (B) The 1.8-kb fragment $\left(D C P_{1-1817}\right)$ regulates expression in the hindgut. $(C)$ The $\mathrm{D}_{1-871}$ fragment does not confer reporter activation. $(D, E)$ The fragments $\mathrm{C}_{872-1309}$ and $\mathrm{P}_{1310-1817}$ direct lac $Z$ expression in the hindgut, weaker than DCP, but in the same spatial pattern. The unspecific lac $Z$ expression in the salivary glands was not observed when using the HZ50PL reporter (data not shown). (F) Immunodetection of Byn protein. The hkbGAL4 directs ectopic Byn expression from the UASbyn transgene in the midgut primordia and the dorsal foregut (between arrows). (G) Antibody stainings for Otp reveal that Byn activates ectopic otp expression in the foregut roof of $h k b G a l 4 /$ UAS-byn embryos (arrowhead).

hindgut tube close to the pads, and is not found in the small intestine of the hindgut (data not shown). Thus, the element contains all the information for proper regulation of $o t p$. A further subdivision of the 1.8 - $\mathrm{kb}$ element yielded three regulatory fragments: a proximal, a central, and a distal. The proximal $\mathrm{P}_{1310-1817}$ and the central $\mathrm{C}_{872-1309}$ fragment were sufficient to direct hindgut-specific lac $Z$ expression in an otp-specific pattern (Fig. $1 \mathrm{D}, \mathrm{E})$, indicating that the gut expression of $o t p$ is regulated by at least two independent Byn-responsive elements. The third, distal fragment $\left(D_{1-871}\right)$ did not give detectable lacZ expression in the embryo (Fig. 1C). 
Byn binds to multiple conserved binding sites within the orthopedia upstream regulatory region

Does Byn directly regulate otp expression via the $\mathrm{C}_{872-1309}$ and the $\mathrm{P}_{1310-1817}$ fragments and what do possible Byn binding sites look like? To address these questions, we performed DNase I protection experiments and found three protected regions in the $\mathrm{C}_{872-1309}$ and two in the $\mathrm{P}_{1310-1817}$ fragment (Fig. 2A). Three of these regions consist of two binding sites, which are arranged in tandem repeats (viii/ix, x/xi) or as nested inverted repeats (xiva and xivb). DNA around the repeats (e.g., the entire region between the binding sites xi and xii) became more sensitive to DNase I in the presence of Byn protein (Fig. 2A). Such hypersensitive sites indicate a compression of the major groove due to DNA bending and suggest a loop formation between the sites.

The comparison of the protected regions in the fragments $\mathrm{P}_{1310-1817}$ and $\mathrm{C}_{872-1309}$ identifies a consensus sequence for Byn binding (Fig. 2B). This consensus of (A/ $\mathrm{G})(\mathrm{A} / \mathrm{T})(\mathrm{A} / \mathrm{T}) \mathrm{NTN}(\mathrm{A} / \mathrm{G}) \mathrm{CAC}(\mathrm{C} / \mathrm{T}) \mathrm{T}$ is similar to the consensus for Brachyury binding (Kispert and Herrmann 1993; see also Fig. 2B). Astonishingly, we also found seven regions within the $\mathrm{D}_{1-871}$ fragment that were protected by Byn from DNase I (Fig. 2B; data not shown). Because $\mathrm{D}_{1-871}$ does not confer lac $Z$ expression in embryos (Fig. 1C) and each of these sites deviates from the consensus sequence of the sites in the $\mathrm{P}$ and $\mathrm{C}$ fragments in at least one position, we conclude that they must be significantly less efficient for transcriptional activation by Byn. These sites probably have low affinity to Byn as suggested by their deviations from the consensus.

Next we investigated whether the Byn sites and their arrangement or spacing are conserved. For this purpose, we compared the sequences of the otp loci of Drosophila melanogaster and Drosophila virilis. We found that seven of the Byn binding sites and their flanking sequences are highly conserved between the two species; only their spacing is somewhat different (Fig. 2C,D). Instead of two Byn tandem sites (viii/ix, x/xi), in D. virilis only two individual sites with spacing similar to the tandems in D. melanogaster are found. Also most of the sites in fragment D are not strictly conserved, although the total number of Byn sites in D. virilis (14; data not shown) is about the same as in D. Melanogaster (15). The large conserved regions flanking the Byn sites are $\mathrm{A} / \mathrm{T}$ rich and presumably facilitate DNA-bending, but do not appear to contain obvious binding sites for other factors. Thus, the high number of binding sites and their embedding in flexible DNA stretches are a conserved feature of the otp regulatory region.

Byn activates orthopedia expression in a synergistic fashion

To test whether Byn is sufficient for otp activation, we performed transactivation assays in Drosophila S2 cells using luciferase as reporter. The basal construct was generated by fusing the $h s p^{70}$-TATA box to the luciferasecoding region. The $1.8-\mathrm{kb}$ element and the three smaller fragments were cloned into the reporter construct and introduced into S2 cells, with and without the pPAc-byn effector plasmid. For the luciferase reporter with the complete 1.8 -kb element $\mathrm{DCP}_{1-1817}$, we observed a 60 fold increase of luciferase activity when Byn was coexpressed (Fig. 3A, supercoiled). This demonstrates that Byn can strongly activate otp expression in S2 cells. However, the fragments $\mathrm{P}_{1310-1817}$ and $\mathrm{C}_{872-1309}$ did not confer any luciferase up-regulation (data not shown). We reasoned that the tension of the supercoiled reporter plasmids might interfere with possibly necessary interactions between the binding sites on the fragments. Indeed, when the $\mathrm{P}_{1310-1817}$ and the $\mathrm{C}_{872-1309}$ reporter cassettes were released from the vector backbone, luciferase transcription was strongly stimulated on Byn coexpression (Fig. 3A). As already seen for the embryo, the $\mathrm{C}_{872-}$ 1309 fragment (165-fold) was more potent than the $\mathrm{P}_{1310-}$ 1817 fragment (130-fold). The relaxed $\mathrm{DCP}_{1-1817}$ reporter conferred an almost sixfold higher transcriptional stimulation than the supercoiled form (Fig. 3A; DCPluc). When placing the $\mathrm{DCP}_{1-1817}$ fragment downstream of the reporter or inverting it to its normal orientation, luciferase stimulation was unchanged, i.e., the fragment truly acts as an enhancer, independent of orientation and location (Fig 3A; luc-DCP; data not shown). As already seen in the embryo for $\beta$-galactosidase (Fig. 1B), the $D_{1-871}$ fragment did not exert any luciferase up-regulation in S2 cells on its own (Fig. 3A), corroborating the notion that the sites might have an insufficient affinity to Byn. However, the combination of fragments C and D stimulated as strongly as the complete DCP reporter (DC-luc; Fig. 3A). Therefore, the sites in D act synergistically with those in $\mathrm{C}$ and are required to reach the full level of $o t p$ transcription. Such a function would explain their evolutionary conservation.

The CP fragment also caused a stimulation as strong as observed for the $P C D$ reporter (Fig. 3A) demonstrating that the combination of Byn sites in $\mathrm{P}$ and $\mathrm{C}$ causes an additive effect on transcription. Byn apparently achieves the appropriate level of transcriptional activation through the action of multiple binding sites, which cooperate, in part synergistically, in part additively.

To confirm that the cotransfection assays reflect the in vivo situation, we tested, in a transient assay, the various luciferase reporters in Drosophila embryos. The reporters were injected into the posterior pole at blastoderm stage where byn is expressed, and the luciferase activity was measured $\sim 16 \mathrm{~h}$ later (Fig. 3B). A high activity was observed when the complete PCD fragment was present in the reporter, and the three individual fragments $\mathrm{P}, \mathrm{C}$, and $\mathrm{D}$ gave the same relative transcriptional activity as in cell culture. No luciferase expression was found for D, C conferred a fraction of the activity directed by the complete $\mathrm{PCD}$ fragment, and $\mathrm{P}$ gave a somewhat weaker transcriptional activity than $\mathrm{C}$. These findings show that levels of Byn protein as ordinarily found in the embryo, are sufficient to activate transcription from the reporter constructs. They confirm that the data achieved in S2 cells are likely to reflect the endogenous regulation of otp by Byn. 
A
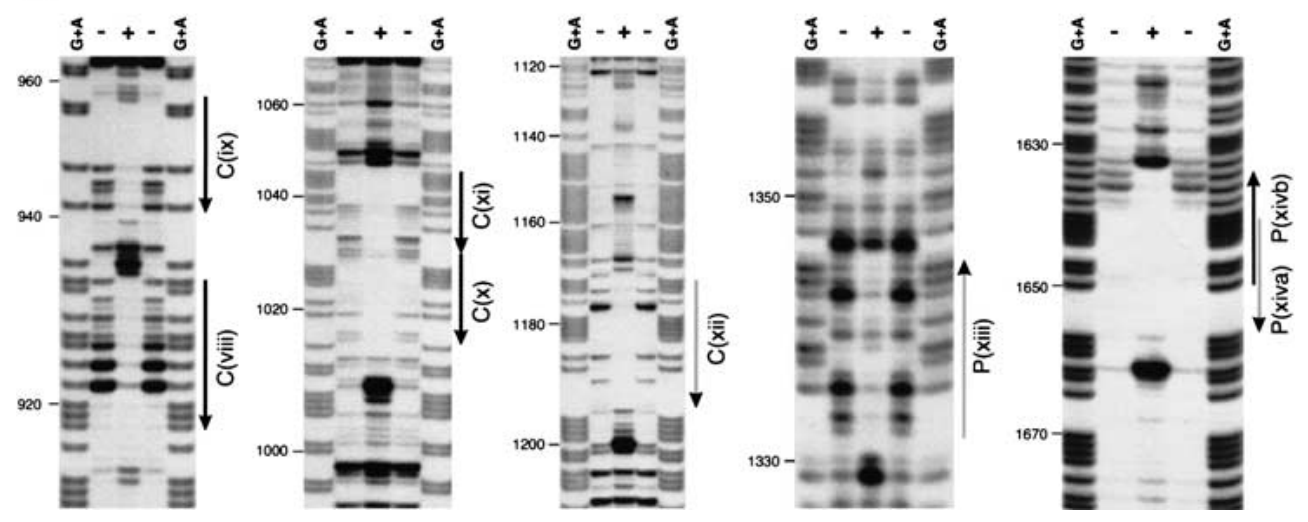

\section{B}

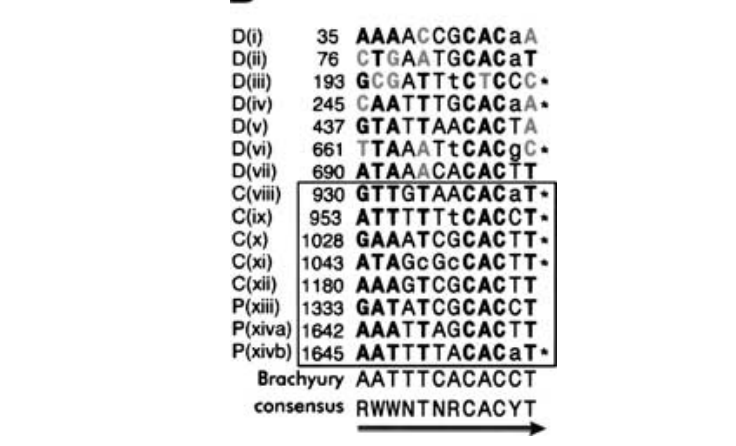

D

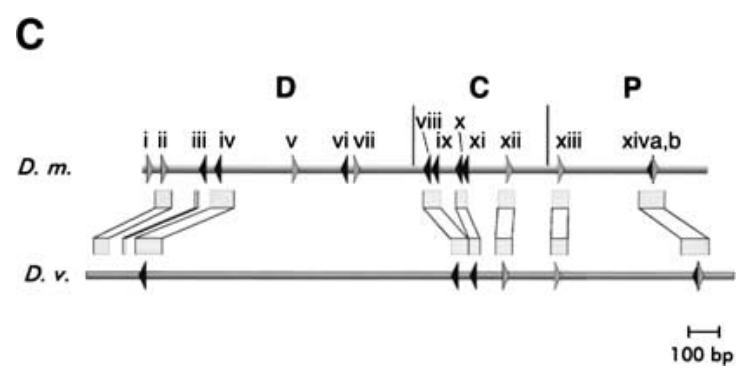

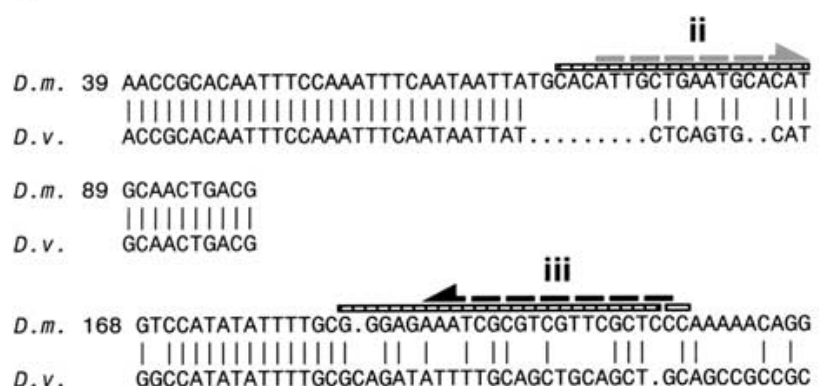

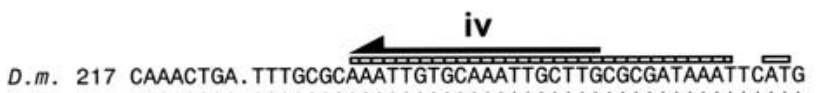

| 1||||||||||||||||||||||||||||||||||||||||||||||

D.v. CAAACTGATTTTGCGCAAATTGTGCAAATTGCTTGCGCGATAAATTCATG

D.m. 266 TTTGCCAATTTTAATTAATTAATCGCTTTGTGCGGTTTTCAT

D.v. TTTGCCAATTTTAATTAATTAATCGCTTGGGGGTTTTCGT

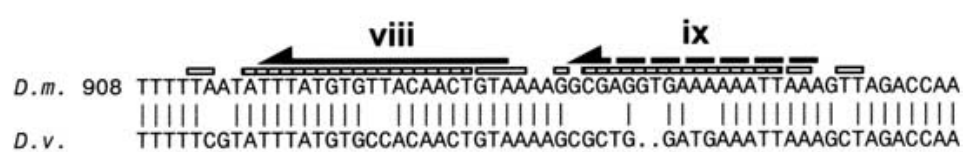

Figure 2. Byn binding sites within the hindgut-specific regulatory region of the otp gene. (A) DNase I protection assays on the $\mathrm{C}_{872-1309}$ and the $\mathrm{P}_{1310-1817}$ fragments. Arrows: orientation of the binding sites as given in $B .(-,+)$ DNase I treatment in the absence and presence of Byn protein, respectively; $(\mathrm{G}+\mathrm{A})$ purine sequence ladder. (B) Sequence of mapped Byn binding sites. The boxed binding sites viii to xivb lie in the functional fragments $\mathrm{C}$ and $\mathrm{P}$. These sites were used for the definition of the Byn binding site consensus. Binding sites within $\mathrm{D}_{1-871}$ (i to vii) deviate from the consensus in up to three positions (gray characters). Brachyury: half of the synthetic palindromic Brachyury binding site (Kispert and Herrmann 1993). (Y) Pyrimide; (R) purine; (W) adenine or thymidine. The numbers indicate the first base of the respective binding site. $\left({ }^{\star}\right)$ Antiparallel strand is listed. (C) Schematic alignment of the hindgut-specific regulatory regions in the otp genes of D. melanogaster (D.m.) and $D$. virilis (D.v.). Boxed regions are $>98 \%$ identical. The triangles represent Byn sites (see B). (Gray triangle) Site in the direction of $o t p$ transcription; (black triangle) site on the complementary strand. Only sites within

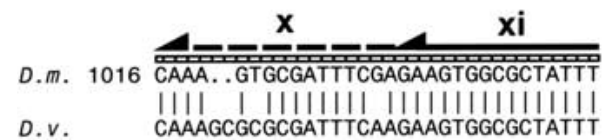

xii

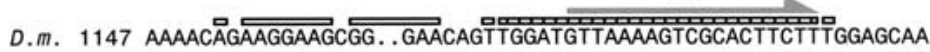
| | | | || || || || | | ||| || ||| || || || || || || || || || | ||| D.v. ATAGCTGAAGGAAGCAGCAGCACACCTGAATGCTAAAAGTCGCACTTCTTGGCTCCAA

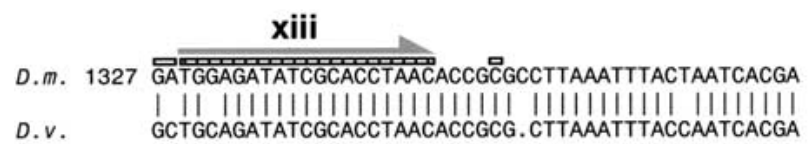
xivb xiva

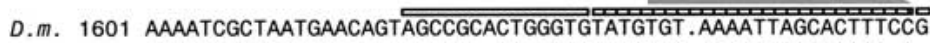
|||||||||||||||||||||||||| ||||||||||||||| ||||||||||||||| | D.v. AAAATCGCTAATGAACAGTAGCCGCGCTGGGTGTATGTGTAAAAATTAGCACTTTGCT

D.m. 1658 GGCAGCATTTTAAAA......... GCAACAACTACAA

D.v. $\quad|\||\||||\||\||\||||\||\||\||\|\mid\|$

conserved stretches are indicated for D. virilis. $(D)$ Alignment as in C. (Black arrows) Byn sites in the direction of otp transcription; (gray arrows) Byn sites on the complementary strand; (hatched rectangles) protected in foot-print; (open rectangles) hypersensitive to DNase I. GenBank accession nos. are AJ306635 (D.m.) and AJ306636 (D.v.). 
A in S2 cells

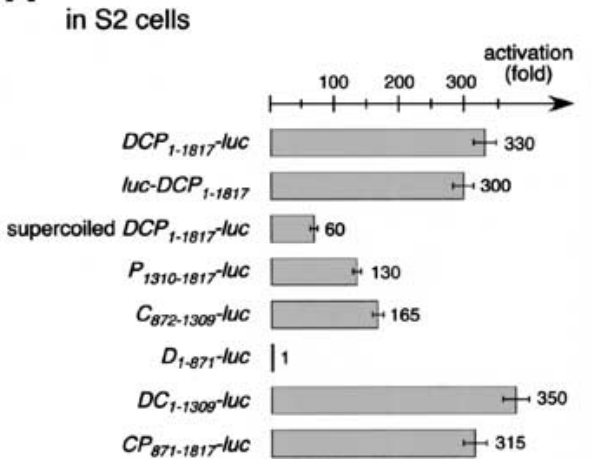

B

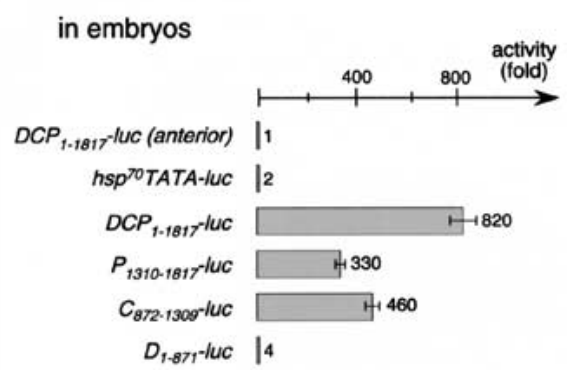

C

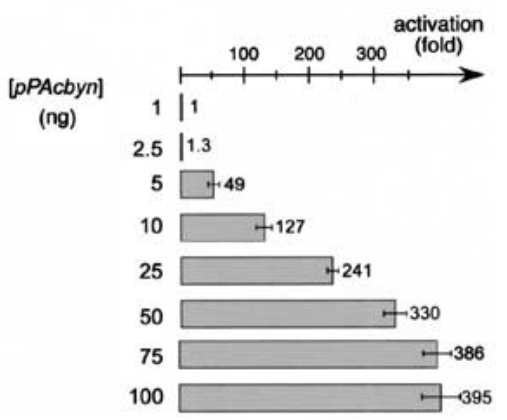

Figure 3. Byn activates otp expression in a synergistic fashion. $(A)$ Relative luciferase activity in cell lysates after transfection of the given constructs with and without the pPAcbyn-effector. A 330 -fold increase of luciferase activity is mediated by the 1.8 -kb $D C P$ reporter $\left(D C P_{1-1817-l u c)}\right.$. The same stimulation is observed when DCP is placed downstream of the luciferase gene (luc-DCP $\left.1-1817\right)$. The luciferase activity is increased only 60-fold when using a supercoiled reporter (supercoiled). $\mathrm{C}_{872-1309}$ stimulates luciferase expression by factor $165, \mathrm{P}_{1310-1817} 130$-fold. $\mathrm{D}_{1-871}$ is not functional on its own. luciferase expression is stimulated 350 -fold when $\mathrm{D}$ is combined with $\mathrm{C}\left(\mathrm{DC}_{1-1309}\right)$. The fusion of $\mathrm{C}$ and $\mathrm{P}\left(\mathrm{CP}_{871-1817}\right)$ results in 315-fold up-regulation. Error bars show the deviations from three experiments after normalization. The reporter constructs were released from the plasmid backbone except for supercoiled. $(B)$ Endogenous levels of Byn protein are sufficient to activate transcription of the reporter constructs. Linearized luciferase reporters were injected into embryos. Depicted is the relative luciferase activity from the lysate of 25 embryos injected with buffer and the given construct, respectively. byn is only expressed in the posterior pole of the embryo, and injection of the $D C P$-reporter into the anterior pole (anterior) does not yield increased luciferase activity. The $h s p^{70} \mathrm{TATA}$-construct only gave marginal expression when injected into the posterior pole (data not shown). When the DCP-reporter is injected into the posterior pole, luciferase activity was increased 820 -fold. The D, C, and P fragments behave as in the cotransfection assay. D does not stimulate, whereas C gives $56 \%$, and P yields $40 \%$ of the activity of DCP. (C) Dose-dependent activation of otp by Byn. S2 cells were transfected with the 1.8otppluc reporter and concentrations of pPAcbyn as indicated in ng. Above a critical concentration of $2 \mathrm{ng}$ the transcriptional stimulation raises in a sigmoidal fashion. Saturation effects are observed at concentrations $>50 \mathrm{ng}$.

Our findings suggest that the Byn dose might be crucial for the levels of target gene activation. To address this question we cotransfected S2 cells with the $1.8-\mathrm{kb}$ $D C P-l u c$ reporter and increasing concentrations of the pPAcbyn effector (Fig. 3C). Intriguingly, activation is only observed above a certain threshold concentration of the effector $(>2.5 \mathrm{ng}$, see Fig. $3 \mathrm{C})$, suggesting that a minimal set of Byn sites must be occupied for activation. At a fourfold higher effector concentration (10 ng) transcriptional activity has reached a 100-fold higher level, supporting the notion of a cooperativity of Byn proteins. Above that, the transcriptional levels reach a plateau, certainly determined by the amount of reporter plasmid.

\section{Single Byn binding sites are essential for transcriptional activation}

We then tested whether the Byn binding sites in the otp regulatory region are relevant for transcriptional activation. This was suggested by the DNase protection, the cotransfection assays, and by their evolutionary conservation. We therefore mutagenized nucleotides of site xii within the $\mathrm{C}$ fragment, which we assumed to be of higher relevance than the other four sites in $\mathrm{C}_{872-1309}$ (see Figs. 2, 4A). xii is not part of a tandem repeat, is inverted to the other Byn sites in the fragment, and is very similar in sequence to the Brachyury consensus half site (Figs. 2B, 5D). When nucleotides were exchanged within site xii, which were protected by Byn in the foot- printing assay, but were not part of the consensus, the binding and protection by Byn was entirely unaffected (Dxii-1, Fig. 4A,B). As expected, these changes did not influence the transcriptional activation by the $\mathrm{C}$ fragment in S2 cells nor in embryos (Fig. 4C-E). When positions within the consensus were changed, Byn was still able to specifically recognize what was left of site xii as revealed by DNase I protection assays ( $\Delta$ xii- 2 to $\Delta$ xii-4; Fig. 4A,B). The specific recognition of the target site as a whole apparently does not depend on a few bases, a notion which is compatible with the significant variability of the Byn sites in otp. However, the base exchanges in the mutant forms $\Delta$ xii-2 to $\Delta$ xii-4, within the consensus, clearly reduced protection against DNase I by Byn, particularly in the mutated regions themselves, demonstrating that the affinity to Byn is significantly reduced (Fig. 4B). Thus, the number of contacts determines the stability of the Byn-DNA interaction. Because site xii is the only single site in the $\mathrm{C}$ fragment, a reduction of the affinity to Byn might drastically affect the functionality of the entire fragment. Indeed, none of the reporter constructs with mutations within the consensus showed transcriptional activation by Byn in cell culture or was active in the embryo (Fig. 4C-E) underlining the relevance of the consensus for Byn function.

The remaining four tandem sites in the $\mathrm{C}$ fragment were not able to confer any transcriptional activity in Schneider cells or the embryo (Fig. 4C-E). To determine their relevance, we mutated each of these sites and 

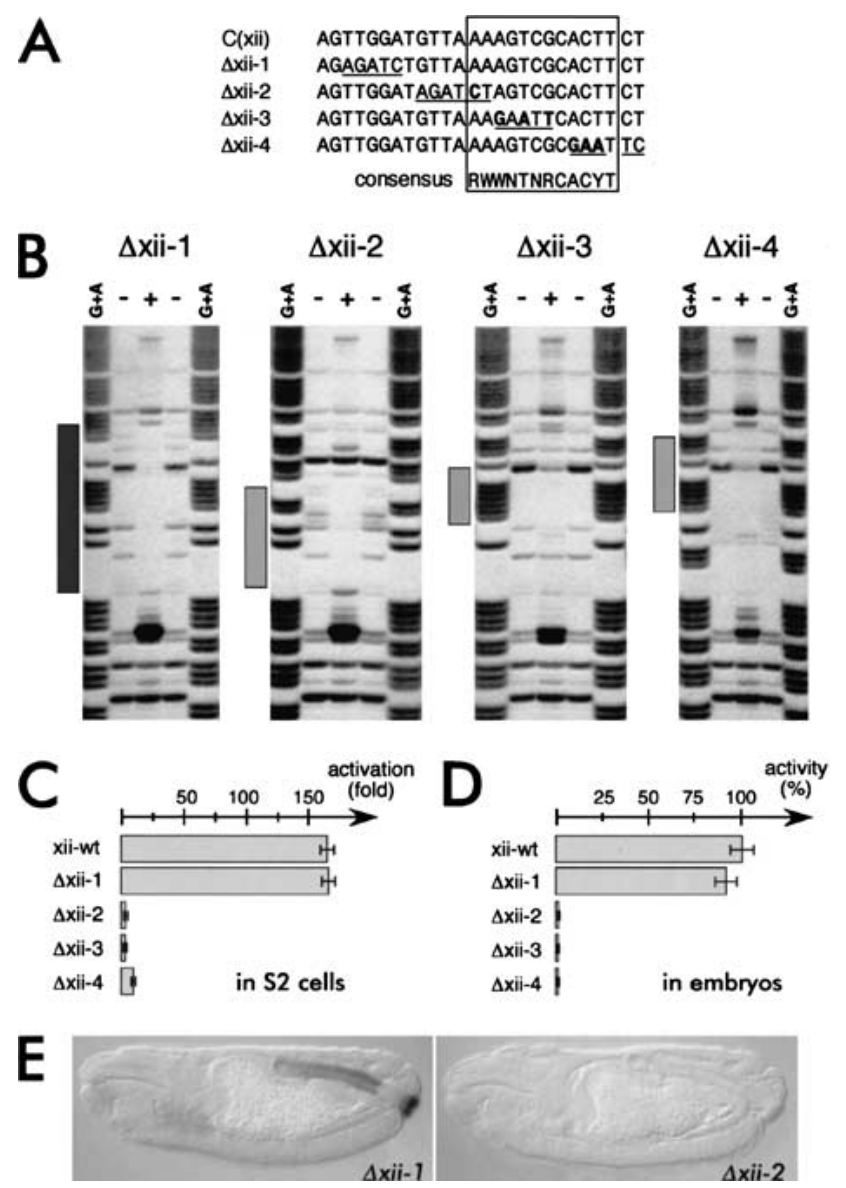

Figure 4. Mutations of Byn sites do not abolish the recognition, but reduce the Byn affinity and abolish the activity in vivo. (A) Mutations induced in site xii within fragment $\mathrm{C}_{872-1309}$. Shown is the DNase-I protected region as shown in Figure 2A. The Byn consensus is boxed. Changes are underlined; bold letters indicate deviations from the consensus. $(B)$ DNase I protection assays on the fragments $\Delta$ xii- 1 to $\Delta$ xii- $4 .(+,-)$ DNase I treatment in presence and absence of Byn, respectively. (G+A) Purine marker ladder. ( $\Delta$ xii-1) The Byn protection pattern is unchanged (dark gray bar, cf. Fig. 2A). ( $\Delta$ xii-2, $\Delta$ xii-3, $\Delta$ xii-4) Byn recognize the changed site, but does not protect the mutated regions (light gray bars, regions of residual protection). $(C, D)$ Mutations within the consensus ( $\Delta$ xii2 to $\Delta$ xii-4) abolish the ability of fragment $\mathrm{C}$ to activate Byn-dependent transcription. (C) Mutated luciferase reporters were tested in cotransfection assays in S2 cells (transcriptional activities are given in comparison to assays without Byn cotransfection). (D) Mutated luciferase reporters were injected into the posterior pole of embryos and were tested for luciferase activity (given as percentage of wild-type xii-wt). (E) Mutations within site xii abolish lacZ activation through the $\mathrm{C}$ fragment in the embryo. Embryos carrying mutated lac $Z$ reporters were immunostained for $\beta$-galactosidase. Changes $5^{\prime}$ to the consensus $(\Delta x i i-1)$ do not affect the

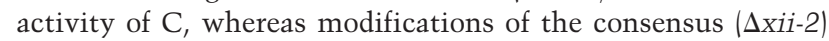
disrupt the functionality of the $l a c Z$ reporter.

tested the resulting $\mathrm{C}$ fragment variants in reporter assays (Fig. 5). The deletion of each site gave a loss of transcriptional stimulation in cell culture (Fig. 5B) and in embryos (Fig. 5C). This shows that all four sites, even though they are arranged in tandems, are relevant for the function of the $\mathrm{C}$ fragment, albeit to a variable degree. For instance, the mutation of site $\mathrm{x}$ led to an $80 \%$ loss in luciferase activity, suggesting that this site represents a high affinity site. Indeed, site $\mathrm{x}$ is very similar to the strictly relevant site xii (Fig. 5D), and we termed these important sites as type A sites. Mutations in sites viii, ix, or xi have much less influence on the transcriptional activation, suggesting that the sites have lower affinity to Byn. These sites have a more relaxed consensus and are tentatively grouped as type B. They share the relaxed consensus with the seven Byn sites in fragment D which are also not sufficient to activate otp transcription. In the $\mathrm{P}$ fragment, a type A Byn site is present as in the C fragment: The single site xiii is very similar to the sites $\mathrm{x}$ and xii, and a truncation of $\mathrm{P}$ eliminating site xiii results in a complete loss of lacZ expression in the embryo (data not shown).

Taken together, the above observations suggest that the minimal Byn response element that confers biological activity consists of one type A site and a set of type B sites. Because site $\mathrm{x}$ is ineffective in conjunction with the sites vii, ix, and $x i$, we assumed that the orientation of the sites towards each other determines whether they are able to stimulate transcription. To test this hypothesis, we generated a set of minimal cis regulatory elements containing various combinations of the type A site xii and the type B site ix and measured their Byndependent transcriptional activity in luciferase reporter assays (Fig. 5E). One single high affinity site xii was not sufficient for the activation of the reporter, whereas the tandem repeat of two sites caused a significant up-regulation of the luciferase activity (23-fold, Fig. 5E, left panel). The combination of two type A site tandem repeats resulted in a somewhat stronger transcriptional stimulation (37-fold), suggesting that the tandem repetition confers an additive effect. Intriguingly, a palindromic arrangement of two type A sites was much more potent than the tandem repeat (48-fold vs. 23 -fold activation), and the combination of two palindromes showed synergistic effects (134-fold stimulation). These data are in general agreement with the properties observed for the synthetic Brachyury site (Kispert et al. 1995).

As expected from the systematic mutations of the sites in fragment $C$, neither one single type B site ix (Fig. $5 \mathrm{E}$, right panel) nor a type $\mathrm{B}$ tandem were sufficient for luciferase activation (data not shown). Also a palindrome of two type B sites and the triple parallel repeat of one high affinity site and two type B sites were insufficient to stimulate transcription. This explains why the type A site $\mathrm{x}$ in conjunction with the type $\mathrm{B}$ sites viii, ix, and $\mathrm{xi}$ could not confer transcriptional activation in the $\mathrm{C}$ fragment without a functional site xii: The four remaining sites all had the same orientation (Fig. 4C-E). However, when one type B site and one type A site were combined as a palindrome, the luciferase activity was significantly stimulated (19-fold). Intriguingly, the fusion of one type A palindrome and one type B palindrome resulted in a 122-fold increase of luciferase transcription. This shows a clear synergistic effect between the two types of Byn 
Figure 5. Multiple Byn binding sites cooperate for maximal transcriptional activation. (A) The four tandem repeat sites (viii to xi) in $\mathrm{C}_{872-1309}$ were mutated and the resulting $\mathrm{C}$ variants ( $\Delta$ viii to $\Delta \mathrm{xi}$ ) were tested in luciferase reporter assays. (Black triangles) Sites viii to $\mathrm{xi}_{\text {; }}$ (gray triangle) site xii; (open triangles) mutated forms. (B) Luciferase activity in $\mathrm{S} 2$ cells. The mutations of the sites ix and xi led to a mild reduction of the luciferase activity. The mutation of site viii causes a strong reduction of $\sim 45 \%$. The mutation of site $\mathrm{x}$ led to an $80 \%$ loss of luciferase transcription. $(C)$ Qualitatively identical results were obtained when injecting the corresponding reporters into the posterior pole of embryos. (D) Comparison of the high affinity Byn sites $\mathrm{x}$, xii, and xiii. Bold letters indicate identical bases. The three sites have a high homology that is shared by the synthetic Brachyury half-site in 10 out of 12 positions. (E) Synthetic combinations of the type A Byn site xii (gray triangles) and the type B Byn site ix (black triangles) were tested in luciferase reporter assays after releasing the reporter cassettes from the vector backbone. Either at least two type A sites or one type A and one type B site in anti-parallel orientation must be present for reporter gene activation. Palindromes of high-affinity Byn sites are more effective in transcriptional stimulation than tandem repeats. Low affinity sites act strongly synergistic in the presence of high affinity sites. sites and strongly suggests that Byn molecules cooperate on the DNA to activate target gene expression.

Mouse and Xenopus Brachyury activate orthopedia expression in Drosophila

All three type A Byn sites show a high homology to a half of the palindromic Brachyury binding site (Fig. 5D; Kispert and Herrmann 1993). Thus, it is very likely that vertebrate Brachyury proteins can recognize Byn sites
B

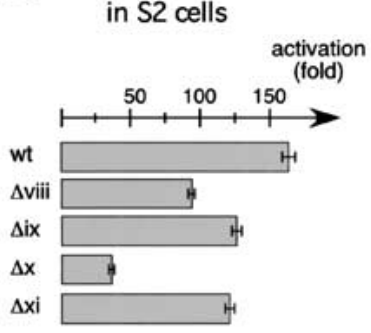

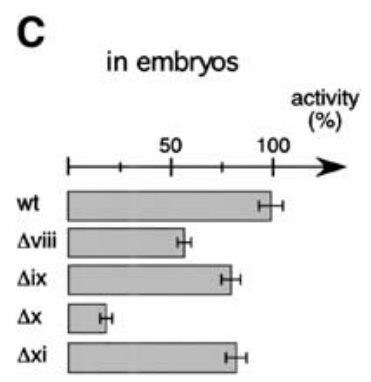

$\mathbf{E}$
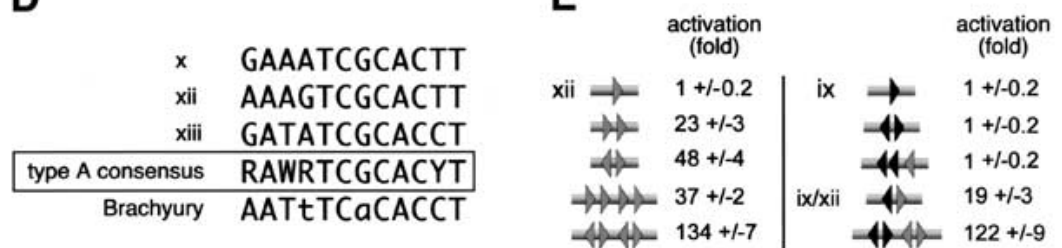
(fold)

ting 
from the 1.8-kb DCP-luc reporter in Drosophila cell culture. As shown in Figure 6E, both Brachyury homologs can activate transcription, although to a lesser extent than Byn, and therefore are apparently able to recognize the Byn sites in the otp gene.

\section{The $T$ domain is necessary and sufficient for the} dimerization of Brachyury-type transcription factors

Byn binding sites of putatively low affinity (type B, on fragment D) exert synergistic effects on target gene expression when combined with putatively high affinity sites (on fragment C; Fig. 3A, DC-luc). Likewise, the synthetic combination of type $\mathrm{A}$ and type $\mathrm{B}$ sites revealed a strong synergism in transcriptional activation (Fig. 5E). Furthermore, extended regions between Byn sites became hypersensitive to DNase I (Fig. 2A) suggesting an interaction between different Byn sites during transcriptional activation, possibly by the physical interaction of Byn itself. The X-ray analysis of Xbra $\mathrm{T}$ domains showed that two Xbra molecules are close enough for a dimerization on the synthetic 24-bp palindrome of the Brachyury consensus (Müller and Herrmann 1997). However, this contact in the crystal could be attributable to the two inverted binding half sites in the palindrome rather than being a biologically relevant dimerization. Therefore, we wished to test a possible Byn-Byn interaction in a cellular context, and fused the Byn open reading frame (ORF) to the activation (GAD-Byn) and to the DNA binding domain (GBD-Byn) of yeast GAL4, respectively. The constructs were introduced into an appropriate yeast strain and, in case of a Byn-Byn interaction, should allow growth on a selective medium due to the formation of a functional GAL4 activator. In fact, combinations of constructs containing the Byn $\mathrm{T}$ domain (amino acids 84-276) were able to confer growth when combined in the cells (Fig. 7A,B), supporting that Byn proteins interact via their DNA-binding domain. To narrow down the interaction region, we generated $\mathrm{N}$ and C-terminal deletions of the Byn $\mathrm{T}$ domain. These truncations revealed that the central region of the $\mathrm{T}$ domain (amino acids 151-205) is sufficient for the interaction. This region contains a stretch of amino acids that forms most of the contact surface of the two Xbra T domains in the crystal (Müller and Herrmann 1997; Fig. 7A), and therefore most likely is also being used for dimerization in vivo. The conservation of this region of the Brachyury-type $\mathrm{T}$ domain suggested that all members of the Brachyury subgroup can dimerize. Indeed, a murine Brachyury-GAL4 fusion construct could interact with the Drosophila Byn interaction domain (Fig. 7), indicating that the ability to dimerize is a general feature of Brachyury proteins.

\section{Discussion}

Brachyury proteins bind to a nonpalindromic recognition motif

We characterized the cis-acting region of Drosophila otp conferring hindgut-specific gene expression (Fig. 1).
A

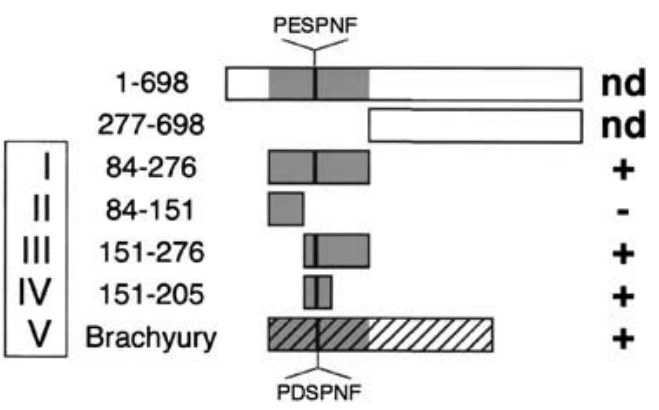

B

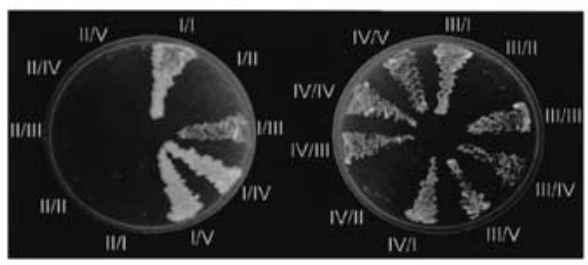

C

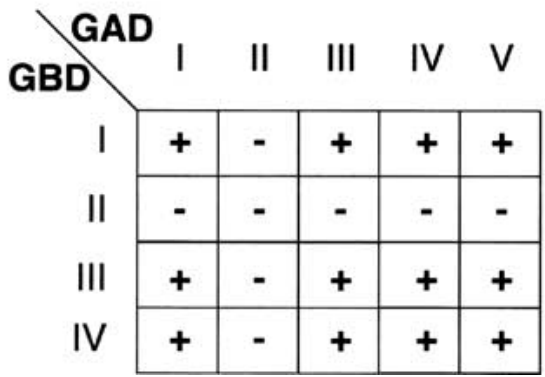

Figure 7. The central portion of the $\mathrm{T}$ domain is necessary and sufficient for the dimerization of Brachyury-type transcription factors in yeast two-hybrid assays. (A) Schematic outline of the constructs tested. Numbers indicate amino acids of Byn (fulllength protein, 698 amino acids). The roman numbers designate the constructs used in $B$. PDSPNF: interaction surface of the crystallized Xbra T domains (Müller and Herrmann 1997); PESPNF: corresponding stretch in Byn. (nd) Interaction not tested; (+/-) ability to dimerize. (B) Yeast cells were grown on adenine-free minimal medium. All constructs containing the PESPNF motif allow growth on selective medium. A mouse Brachyury-GAD fusion construct interacts with all those GBDByn constructs containing the PESPNF motif. (C) Schematic representation of the interactions shown in $B$.

Within a $1.8-\mathrm{kb}$ regulatory element we identified 15 binding sites for Brachyenteron that have the consensus sequence $(\mathrm{A} / \mathrm{G})(\mathrm{A} / \mathrm{T})(\mathrm{A} / \mathrm{T}) \mathrm{NTN}(\mathrm{A} / \mathrm{G}) \mathrm{CAC} / \mathrm{C} / \mathrm{T}) \mathrm{T} \quad$ (Fig. $2 B)$. This consensus matches the half-site of the previously characterized palindromic recognition consensus for mouse Brachyury (Kispert and Herrmann 1993). In fact, mouse Brachyury could activate otp in the Drosophila embryo and therefore is able to recognize Byn target sites (Fig. 6). Several elements in the consensus appear to contribute to the recognition of a site by Byn. The mutation of some bases, which left other parts of the consensus intact, did not abolish binding entirely (Fig. 4). However, a Byn site requires an intact consensus to function in transcriptional activation.

Surprisingly, in the regulatory region of otp we did not find a single Byn site that would be sufficient to activate 
transcription on its own (Fig. 5E). Moreover, we also did not find a palindromic site corresponding to the one identified for Brachyury (Kispert and Herrmann 1993). xiva and xivb are antiparallel to each other, somewhat resembling a palindrome, but they overlap so much that we assume that the binding of Byn to one of the two sites might disturb the binding to the other.

Also in other cases in which natural target sites for $\mathrm{T}$ domain transcription factors have been described, only single half-sites were identified by virtue of their high similarity to the original Brachyury consensus. For Xbra, recognition motifs matching the Byn consensus have been found in the efgf locus (Casey et al. 1998). Also the $\mathrm{T}$ domain proteins Tbx-2, Mga, and VegT, Tpit recognize binding motifs similar to high affinity Brachyury halfsites (Carreira et al. 1998; Tada et al. 1998; Hurlin et al. 1999; Lamolet et al. 2001). Thus, rather than to a palindrome Brachyury, proteins seem to bind a 12-bp site in vivo. Possibly, the palindrome is even disadvantageous for the animal and might cause an exceedingly stable occupancy of the site by the protein.

Within the regulatory region of otp we have observed an unusually high degree of tolerated variability for Byn binding sites. Within the 15 characterized sites we distinguished two types, A and B. Type A sites (to which the sites $\mathrm{x}$, xii, and xiii belong) have a consensus, which differs only by two positions from the Brachyury consensus (Fig. 5D). This consensus has been selected from DNA molecules that bind optimally to Brachyury by binding site selection (Kispert and Herrmann 1993), a method that enriches for high-affinity binding sites. We therefore assume that the type A sites constitute highaffinity sites for Byn. More importantly, type A sites are essential for strong transcriptional up-regulation. Site $\mathrm{x}$ is required for $80 \%$ of the transcriptional activation by the $\mathrm{C}$ fragment (Fig. 5B,C), site xii is absolutely essential for $\mathrm{C}$ (Fig. 4), and variants of the $\mathrm{P}$ fragment without site xiii lost the ability to activate transcription (data not shown). Finally, two type A sites, in tandem or in inverted orientation, are sufficient to activate Byn-dependent transcription (Fig. 5E).

The Byn binding sites of type B yield a more relaxed consensus that encompasses the high-affinity consensus. They are significantly less efficient to activate Byn-dependent transcription than type A sites (Figs. 1,3,5). For instance, the set of seven type B sites in the D fragment (i to vii) was not sufficient for transcription on its own (Figs. 1C, 3A,B), and the sites ix and xi contribute little to the activity of fragment $C$ (Fig. 5A-C). Based on these observations we propose that the type $\mathrm{B}$ sites constitute low-affinity sites for Byn. In contrast to type A sites, a type $\mathrm{B}$ doublet is not able to activate transcription in the synthetic Byn response elements, neither in tandem nor in inverted orientation (Fig. 5E; data not shown). Only in combination with a type A site, which is inverted to the type B site, is transcriptional activation observed.

What could be the basis for the ability of the Byn $T$ domain to bind to such variable target sequences? Due to the high homology between Xbra and Byn and between their target sites, it is reasonable to assume that the crys- tal structure analysis of the Xbra T domain (Müller and Herrmann 1997) could serve as a model for Byn. The basic residues of the highly conserved motif $\mathrm{CAC} / \mathrm{C} / \mathrm{T}) \mathrm{T}$ are contacted by amino acids of the $\mathrm{T}$ domain and constitute a sequence-specific binding interaction. Nucleotide exchanges within this stretch would disrupt the hydrogen bonds to the protein and lead to significantly lower binding efficiency. This is the case for the variant site $\Delta$ xii-4, which is only marginally protected against DNase by Byn (see Fig. 4B). A number of type A sites do not have the $3^{\prime}-\mathrm{T}$ of $\mathrm{CAC}(\mathrm{C} / \mathrm{T}) \mathrm{T}$, a feature which presumably contributes to their low binding efficiency (see Fig. 3B). At the $5^{\prime}$ end of the Byn consensus the RWWmotif defines a particular conformation of the double helix rather than a specific base sequence. At these positions the $\mathrm{T}$ domain contacts the phosphoribosyl backbone of the nucleotides (Müller and Herrmann 1997), stabilizing the protein-DNA interaction, but allowing a higher variability of the bases. Thus, the structural data for the $\mathrm{T}$ domain give a good explanation how Brachyury proteins can accommodate the variability of their target sequences. It is reasonable to assume that $\mathrm{T}$ domain transcription factors, not belonging to the Brachyurytype subgroup, also recognize a wide range of target sites with variable affinities.

\section{Complexity of the regulatory region}

The number of Byn binding sites in the regulatory region of otp is surprisingly high, but all sites seem to contribute to proper otp expression. In the $\mathrm{C}$ fragment, one site (xii) is absolutely essential, but the other four in the fragment also contribute to transcriptional up-regulation (Fig. 5). The synergism between the sites $\mathrm{x}$ and $\mathrm{xii}$ is remarkable because without site $\mathrm{x}$, site xii gives, though in conjunction with vii, ix and xi, only a low transcriptional activation. However, the expression level induced by $\mathrm{C}$ is only a fraction of the one induced by the entire 1.8-kb fragment (Fig. 3). The sites in $\mathrm{P}$ add to the level achieved by $\mathrm{C}$ and yield, when joined with $\mathrm{C}$, almost the level of the complete fragment (Fig. 3). The contribution of the seven sites in the D fragment is again synergistic rather than additive. Their activity is noticeable when comparing the transcriptional activity of the $\mathrm{C}$ fragment to the one of DC (Fig. 3). The sites in D bring C up to wild-type level although they have no activity on their own (Fig. 3). Presumably, they are occupied in the cell only when high-affinity type A sites like those in $\mathrm{C}$ are nearby. We suggest that the entire set of binding sites cooperates to achieve a particular level of target gene expression in the embryo.

Also for the regulation of efgf by Xbra, several Xbrabinding sites seem to cooperate. Only two Xbra sites had been identified by virtue of their sequence homology to the ideal Brachyury half-site (Casey et al. 1998). However, more potential binding sites, some of them in tandem, can be found within the $f g f$ loci of frog, mouse, and human when the Byn consensus is scored (data not shown). Truncations of the efgf upstream regulatory regions caused the stepwise reduction of XBra-dependent 
reporter gene expression (Casey et al. 1998), and these reductions correlate with the loss of potential Xbra sites matching the Byn consensus. Thus, also for the Xbra target gene efgf, multiple binding sites appear to be relevant to reach a certain level of expression. We propose that such complex regulatory regions with multiple sites are a general feature of target genes for Brachyury transcription factors.

\section{Brachyenteron binds to DNA cooperatively}

We have the clear indication that different Byn sites in the regulatory region of otp functionally interact, for instance those of the $\mathrm{D}$ and the $\mathrm{C}$ fragment and those within the $\mathrm{C}$ fragment. We propose that contacts between Byn proteins on different sites mediate this interaction physically. Several lines of evidence support this notion. Long stretches of DNA between Byn sites become hypersensitive to DNase I in the presence of Byn protein (Fig. 2A), indicating a loop formation between these sites. The A/T-repeat rich sequences flanking the Byn sites are evolutionary conserved (Fig. 2C) and might act as hinges between them, thereby facilitating DNAbending by Byn. The relaxation of the luciferase reporters caused a considerable increase of Byn-mediated transcription (Fig. 3), supporting that Byn-Byn interactions on the DNA are indeed important for the transcriptional activation. In addition, the synergism of type A and type $\mathrm{B}$ sites in the synthetic Byn response elements strongly suggest a stabilization of Byn-DNA interactions at low affinity sites in the presence of high affinity sites (Fig. $5 E)$. Finally, we could show that two Byn molecules interact via a conserved stretch of the $\mathrm{T}$ domain in the two-hybrid assay (Fig. 7). This region included the contact surface, which has been identified for the Xbra T domains in the crystal structure (Müller and Herrmann 1997), PDSPNF for vertebrate Brachury proteins, PESPNF for Byn. We have evidence that Brachyury-type transcription factors associate through this surface to form homodimers. We do not know anything about the specificity or potential promiscuity of the interaction, but mouse Brachyury could interact with the Byn $\mathrm{T}$ domain. Also other types of $\mathrm{T}$ domain transcription factors have a similar stretch of amino acids in the $\mathrm{T}$ domain with AT or RS instead of the NF within the hexapeptide. They can probably homodimerize as well, but it remains to be seen whether the molecules are also able to form heterodimers within the family.

\section{A mechanism for dose-dependent target gene regulation by Brachyury proteins?}

We have shown that Byn binds to different types of target sites cooperatively and thereby activates a certain level of otp expression. Particular arrangements of such highand low-affinity sites could also serve another purpose: the dose-dependent activation of target genes. There are several indications in Drosophila that the dose of Byn is relevant and informative. Byn is expressed in a dorso- ventral gradient in the blastoderm (T. Kusch and $\mathrm{R}$. Reuter, unpubl.) and allelic series of byn mutants exhibit graded defects in the embryos (Singer et al. 1996; Kusch and Reuter 1999|. This is fully consistent with the finding that above a certain threshold of Byn effector, the cis-regulatory region of otp strongly responds transcriptionally (Fig. 3C). In other species, Brachury-type transcription factors also give very clear dose-dependent responses. In vertebrates, Brachyury is distributed in an antero-posterior gradient in the embryo, and the formation of distinct embryonic structures depends on certain thresholds of Brachyury (MacMurray and Shin 1988; Stott et al. 1993; O'Reilly et al. 1995). It is conceivable that target gene responses depend on the levels of Brachyury expression as well as the amount and combination of high- and low-affinity sites within their regulatory regions. The mechanisms might not be unlike the dose-dependent transcriptional regulation by the Drosophila morphogen Bicoid (Driever and Nüsslein-Volhard 1989; Burz et al. 1998). Bicoid is distributed in an antero-posterior gradient, binds to DNA cooperatively, and distinct Bicoid target genes respond to certain thresholds of the morphogen depending on the composition of high- and low-affinity sites within their regulatory regions.

Other $\mathrm{T}$ domain factors outside the Brachyury-type subfamily also regulate developmental processes in a dose-dependent fashion, such as the Tbx proteins 1-5 controlling the formation of different limb structures (Isaac et al. 1998; Rodriguez-Esteban et al. 1999). Thus, it is likely that a mechanism of dose-dependent target gene regulation that involves the high- and low-affinity sites that we described, is not only true for Brachyury, but also for other $\mathrm{T}$ domain proteins.

\section{Materials and methods}

\section{Generation of recombinant Byn protein}

The expression and purification of C-terminally $\mathrm{His}_{6}$-tagged full-length Byn has been described previously (Kusch and Reuter 1999). The DNA-binding fraction of solubilized Byn was affinity-purified using heparin columns (Hitrap; Pharmacia). The binding fraction was eluted with $0.5 \mathrm{M} \mathrm{NaCl}$ and was dialyzed against DNA-binding buffer (25 mM HEPES at $\mathrm{pH} 7.2,35 \mathrm{mM}$ $\mathrm{NaCl}, 35 \mathrm{mM} \mathrm{KCl}, 10 \%$ glycerol, $2.5 \mathrm{mM} \mathrm{MgCl}_{2}, 0.5 \mathrm{mM} \mathrm{DTT}$, $0.5 \%$ NP-40, $4 \mathrm{mg} / \mathrm{mL} \mathrm{BSA).}$

\section{DNase I protection assays}

otp regulatory modules in pBluescript KS II were endlabeled with $\alpha^{32} \mathrm{P}$-dCTP (Amersham) using Klenow polymerase (Boehringer). For DNase I protection assays, an equivalent of 15,000 cpm was incubated with $0.2 \mathrm{pmol}$ Byn protein in a total volume of $50 \mu \mathrm{L}$ of DNA-binding buffer containing $1 \mu \mathrm{g}$ poly dGdC/poly $\mathrm{dGdC}$ for $25 \mathrm{~min}$ at $25^{\circ} \mathrm{C}$. The reactions were prechilled for 2 min on ice before adding $50 \mu \mathrm{L}$ of DNase I suspension (1:2500$1: 7500$ in $10 \mathrm{mM} \mathrm{MgCl}_{2}, 5 \mathrm{mM} \mathrm{CaCl}_{2}$ ). After $90 \mathrm{sec}$ the reaction was stopped by adding $100 \mu \mathrm{L}$ of stop solution $(200 \mathrm{mM} \mathrm{NaCl}$, $1 \%$ SDS, $200 \mu \mathrm{g} / \mathrm{mL}$ ssDNA, $20 \mathrm{mM}$ EDTA, and $10 \mathrm{mg} / \mathrm{mL}$ proteinase K) and by vigorous mixing for $30 \mathrm{sec}$. An equivalent of $5000 \mathrm{cpm}$ was separated together with the chemically depu- 
rinated fragment $(\mathrm{G}+\mathrm{A}$ ladder $)$ on a $0.2 \mathrm{~mm}$ sequencing gel. The gels were fixed, dried, and exposed on Kodak X-Omat AR films for $18-36 \mathrm{~h}$.

\section{Mutagenesis of Byn binding sites}

Site directed mutagenesis was performed by PCR using mismatch primers, which introduce an additional restriction site. otp regulatory modules in pBluescript KS II (Stratagene) were subjected to a first PCR using a universal primer (T3 or T7; Stratagene) and the appropriate mismatch primer. The resulting fragments were combined as templates for a second PCR with $\mathrm{T} 3$ and T7 primers. Modifications were confirmed by restriction analysis and sequencing. Primer sequences are available on request.

\section{Luciferase reporter assays}

The $h s p^{70}$ TATA box was cloned via BgIII and HindIII into pGL2 (Promega). Fusions of the Byn sites xii and ix were generated using appropriate oligonucleotides. All constructs were sequenced and inserted upstream of the $h s p^{70}$ TATAluc reporter plasmid (detailed information is available on request). The effector plasmids were generated by fusing full-length cDNAs coding for byn, Xbra (a gift of R. Winklbauer, MPI Developmental Biology, Tübingen, Germany), or mouse Brachyury (a gift of A. Kispert, Institute of Molecular Biology, $\mathrm{MHH}$, Hannover, Germany) to the actin $5 \mathrm{C}$ promoter of the $p P A c$ vector (Krasnow et al. 1989). S2 cells from two $60 \mathrm{~mm}$ petri dishes were transfected at a density of $3 \times 10^{6}$ cells $/ \mathrm{mL}$ by means of standard calcium phosphate coprecipitation. For transfection of two dishes, $200 \mathrm{ng}$ of the reporters, $50 \mathrm{ng}$ of $p P A c-b y n$ (if not stated otherwise, Fig. $3 \mathrm{Cl}, 4 \mu \mathrm{g}$ of the $p P A c-l a c Z$ normalization construct (Driever and Nüsslein-Volhard 1989), and $10 \mu \mathrm{g}$ of carrier DNA were used. All transfections were performed at least three times in independent experiments. The medium was changed after $16 \mathrm{~h}$, and the cells were harvested after $24 \mathrm{~h}$. After washing in PBS, the cells were lysed for $15 \mathrm{~min}$ in extraction buffer $(25$ $\mathrm{mM}$ Tris $-\mathrm{H}_{3} \mathrm{PO}_{4}$ at $\mathrm{pH}$ 8.0, $2 \mathrm{mM}$ CDTA, $2 \mathrm{mM}$ DTT, $10 \%$ glycerol, and $1 \%$ Triton X-100). Cellular debris was removed by centrifugation.

The injection of the luciferase reporters into Drosophila embryos was essentially performed as described by Driever and Nüsslein-Volhard (1989). Fifty embryos were injected at blastoderm stage for each assay. Sixteen hours after injection, the embryos were washed with heptane and with PBS, $0.1 \%$ Tween 20. Next, $200 \mu \mathrm{L}$ luciferase lysis buffer were added, and the embryos were devitellinized by sonication $(4 \times 5 \mathrm{sec})$. The embryos were extracted for $15 \mathrm{~min}$ at room temperature. Cellular debris was removed by centrifugation.

Ten percent of cell extracts and $50 \%$ of embryo extracts were used for the luciferase assay. $100 \mu \mathrm{L}$ of luciferase assay buffer [20 $\mathrm{mM}$ Tricine, $1 \mathrm{mM}\left(\mathrm{MgCO}_{3}\right)_{4} \times \mathrm{Mg}(\mathrm{OH})_{2} \times 5 \mathrm{H}_{2} \mathrm{O}$ at pH 7.8, 2.7 $\mathrm{mM} \mathrm{MgSO}_{4}, 0.1 \mathrm{mM}$ EDTA, $30 \mathrm{mM}$ DTT, $250 \mu \mathrm{M}$ LiCoA, 450 $\mu \mathrm{M}$ K-luciferin (Promega), $0.5 \mathrm{mM} \mathrm{Na}_{2}$-ATP] were added to the lysate before measuring (Berthold Lumat LB9501/1 luminometer). Thirty percent of the cell lysate was used for normalization with oNPG as substrate following standard protocols. All enzymatic assays were performed in duplicates.

\section{Fly strains and immunohistochemistry}

For the generation of $l a c Z$ reporters, either the pCAB vector (Bachmann and Knust 1998) or the HZ50PL vector (Hiromi and Gehring 1987) were used. For the generation of the UASmmT transgene the full-length mouse Brachyury cDNA was released from pme75 (a gift of B. Herrmann, MPI Immunobiology, Freiburg, Germany) and introduced into the pUAST vector (Brand and Perrimon 1993) via EcoRI sites. Germline transformation was performed following standard methods. The G455 GAL4-driver line Is(2)P[GAL4]455 was a gift of U. Hinz (Hinz et al. 1994). The huckebein (hkb) GAL4 driver was generated by fusing a proximal 1.2-kb EcoRI-Pst fragment of the $h k b$ promoter region (Häder et al. 2000) to the GawB-vector (Brand and Perrimon 1993). Polyclonal antisera were used for the detection of $E$. coli $\beta$-galactosidase (mouse, Sigma). The Otp antibody was made in rabbits and used at a concentration of 1:200. The anti-Byn antibody and the UAS-byn transgenes have been described previously (Kusch and Reuter 1999). For the detection of mouse Brachyury, the anti- $\mathrm{TN}_{1-123}$ antibody was used (a gift of B. Herrmann; Kispert and Herrmann 1993). Biotinylated secondary antibodies (goat, Jackson Laboratories) and the Vectastain ABC Elite enhancement kit (Vector) were used.

\section{Yeast two hybrid system}

The yeast two-hybrid system has been performed essentially as described previously (James et al. 1996). Briefly, the full-length byn coding region and its fragments were fused with the pGAD and $\mathrm{pGBD}$ vectors (cloning details are available on request). The constructs were introduced into the PJ69-4a yeast strain and the cells were grown on synthetic minimal medium lacking adenine. GBD-Byn fusion constructs containing the C-terminus of Byn could not be used, as this region of Byn already activated the marker gene in the absence of GAD.

\section{Acknowledgments}

We thank G. Brönner, B. Herrmann, U. Hinz, A. Kispert, and R. Winklbauer for reagents; $H$. Thelen and U. 'The Needle' Irion for helping to generate the $h k b G a l 4$ transgenic flies. We are indebted to Drs. N. Berns, M. Carrozza, and R. Schröder for insightful comments on the manuscript. The work was supported by the Fond der Chemischen Industrie and the Deutsche Forschungsgemeinschaft as part of the SFB243 (T.K. and R.R.).

The publication costs of this article were defrayed in part by payment of page charges. This article must therefore be hereby marked "advertisement" in accordance with 18 USC section 1734 solely to indicate this fact.

\section{Note}

During the revision of this manuscript, a paper has been published reporting the identification of a binding site for Tbx5 with high similarity to the described Byn consensus (Hiroi et al. 2001. Nat. Genet. 28: 276-280).

\section{References}

Bachmann, A. and Knust, E. 1998. Positive and negative control of Serrate expression during early development of the Drosophila wing. Mech. Dev. 76: 67-78.

Bassham, S. and Postlethwait, J. 2000. Brachyury (T) expression in embryos of a larvacean urochordate, Oikopleura dioica, and the ancestral role of T. Dev. Biol. 220: 322-332.

Brand, A.H. and Perrimon, N. 1993. Targeted gene expression as a means of altering cell fates and generating dominant phenotypes. Development 118: 401-415.

Burz, D.S., Rivera-Pomar, R., Jäckle, H., and Hanes, S.D. 1998. Cooperative DNA-binding by Bicoid provides a mechanism 
for threshold-dependent gene activation in the Drosophila embryo. EMBO J. 17: 5998-6009.

Carreira, S., Dexter, T.J., Yavuzer, U., Easty, D.J., and Goding, C.R. 1998. Brachyury-related transcription factor Tbx2 and repression of the melanocyte-specific TRP-1 promoter. Mol. Cell. Biol. 18: 5099-5108.

Casey, E.S., O'Reilly, M.A., Conlon, F.L., and Smith, J.C. 1998. The T-box transcription factor Brachyury regulates expression of eFGF through binding to a non-palindromic response element. Development 125: 3887-3894.

Driever, W. and Nüsslein-Volhard, C. 1989. The bicoid protein is a positive regulator of hunchback transcription in the early Drosophila embryo. Nature 337: 138-143.

Häder, T., Wainwright, D., Shandala, T., Saint, R., Taubert, H., Brönner, G., and Jäckle, H. 2000. Receptor tyrosine kinase signaling regulates different modes of Groucho-dependent control of Dorsal. Curr. Biol. 10: 51-54.

Hinz, U., Giebel, B., and Campos-Ortega, J.A. 1994. The basichelix-loop-helix domain of Drosophila lethal of scute protein is sufficient for proneural function and activates neurogenic genes. Cell 76: 77-87.

Hiromi, Y. and Gehring, W.J. 1987. Regulation and function of the Drosophila segmentation gene fushi tarazu. Cell 50: 963-974.

Hurlin, P.J., Steingrimsson, E., Copeland, N.G., Jenkins, N.A., and Eisenman, R.N. 1999. Mga, a dual-specificity transcription factor that interacts with Max and contains a T domain DNA-binding motif. EMBO J. 18: 7019-7028.

Isaac, A., Rodriguez-Esteban, C., Ryan, A., Altabef, M., Tsukui, T., Patel, K., Tickle, C., and Izpisua-Belmonte, J.C. 1998. Tbx genes and limb identity in chick embryo development. Development 125: 1867-1875.

James, P., Halladay, J., and Craig, E.A. 1996. Genomic libraries and a host strain designed for highly efficient two-hybrid selection in yeast. Genetics 144: 1425-1436.

Kispert, A. and Herrmann, B.G. 1993. The Brachyury gene encodes a novel DNA binding protein. EMBO J. 12: 3211-3220.

Kispert, A., Herrmann, B.G., Leptin, M., and Reuter, R. 1994. Homologs of the mouse Brachyury gene are involved in the specification of posterior terminal structures in Drosophila, Tribolium, and Locusta. Genes \& Dev. 8: 2137-2150.

Kispert, A., Koschorz, B., and Herrmann, B.G. 1995. The T protein encoded by Brachyury is a tissue-specific transcription factor. $Е M B O$ T. 14: 4763-4772.

Krasnow, M.A., Saffman, E.E., Kornfeld, K., and Hogness, D.S. 1989. Transcriptional activation and repression by Ultrabithorax proteins in cultured Drosophila cells. Cell 57: 10311043.

Kusch, T. and Reuter, R. 1999. Functions for Drosophila brachyenteron and forkhead in mesoderm specification and cell signalling. Development 126: 3991-4003.

Lamolet, B., Pulichino, A., Lamonerie, T., Gauthier, Y., Brue, T., Enjalbert, A., and Drouin, J. 2001. A pituary cell-restricted T box factor, Tpit, activates POMC transcription in cooperation with Pitx homeoproteins. Cell 104: 849-859.

MacMurray, A. and Shin, H.S. 1988. The antimorphic nature of the Tc allele at the mouse T locus. Genetics 120: 545-550.

Müller, C.W. and Herrmann, B.G. 1997. Crystallographic structure of the T domain-DNA complex of the Brachyury transcription factor. Nature 389: 884-888.

O'Reilly, M.A., Smith, J.C., and Cunliffe, V. 1995. Patterning of the mesoderm in Xenopus: Dose-dependent and synergistic effects of Brachyury and Pintallavis. Development 121: 1351-1359.

Peterson, K.J., Cameron, R.A., Tagawa, K., Satoh, N., and Davidson, E.H. 1999. A comparative molecular approach to me- sodermal patterning in basal deuterostomes: The expression pattern of Brachyury in the enteropneust hemichordate Ptychodera flava. Development 126: 85-95.

Rodriguez-Esteban, C., Tsukui, T., Yonei, S., Magallon, J., Tamura, K., and Izpisua Belmonte, J.C. 1999. The T-box genes $\mathrm{Tbx} 4$ and $\mathrm{Tbx} 5$ regulate limb outgrowth and identity. Nature 398: 814-818.

Shoguchi, E., Satoh, N., and Maruyama, Y.K. 2000. A starfish homolog of mouse T-brain-1 is expressed in the archenteron of Asterina pectinifera embryos: Possible involvement of two T-box genes in starfish gastrulation. Dev. Growth Differ. 42: 61-68.

Simeone, A., D'Apice, M.R., Nigro, V., Casanova, J., Graziani, F., Acampora, D., and Avantaggiato, V. 1994. Orthopedia, a novel homeobox-containing gene expressed in the developing CNS of both mouse and Drosophila. Neuron 13: 83-101.

Singer, J.B., Harbecke, R., Kusch, T., Reuter, R., and Lengyel, J.A. 1996. Drosophila brachyenteron regulates gene activity and morphogenesis in the gut. Development 122: 3707-3718.

Smith, J. 1999. T-box genes: What they do and how they do it Trends Genet. 15: 154-158.

Stott, D., Kispert, A., and Herrmann, B.G. 1993. Rescue of the tail defect of Brachyury mice. Genes \& Dev. 7: 197-203.

Tada, M., Casey, E.S., Fairclough, L., and Smith, J.C. 1998. Bix1, a direct target of Xenopus T-box genes, causes formation of ventral mesoderm and endoderm. Development 125: 39974006.

Technau, U. and Bode, H.R. 1999. HyBra1, a Brachyury homologue, acts during head formation in Hydra. Development 126: $999-1010$.

Wattler, S., Russ, A., Evans, M., and Nehls, M. 1998. A combined analysis of genomic and primary protein structure defines the phylogenetic relationship of new members if the T-box family. Genomics 48: 24-33.

Woollard, A. and Hodgkin, J. 2000. The Caenorhabditis elegans fate-determining gene mab-9 encodes a T-box protein required to pattern the posterior hindgut. Genes \& Dev. 14: 596-603. 


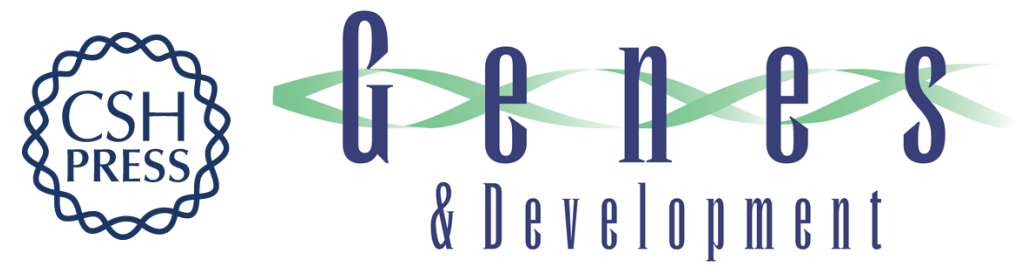

\section{Brachyury proteins regulate target genes through modular binding sites in a cooperative fashion}

Thomas Kusch, Tobias Storck, Uwe Walldorf, et al.

Genes Dev. 2002, 16:

Access the most recent version at doi:10.1101/gad.213002

$\begin{array}{ll}\text { References } & \text { This article cites } 33 \text { articles, } 17 \text { of which can be accessed free at: } \\ \text { http://genesdev.cshlp.org/content/16/4/518.full.html\#ref-list-1 }\end{array}$

License

Email Alerting Receive free email alerts when new articles cite this article - sign up in the box at the top Service right corner of the article or click here.

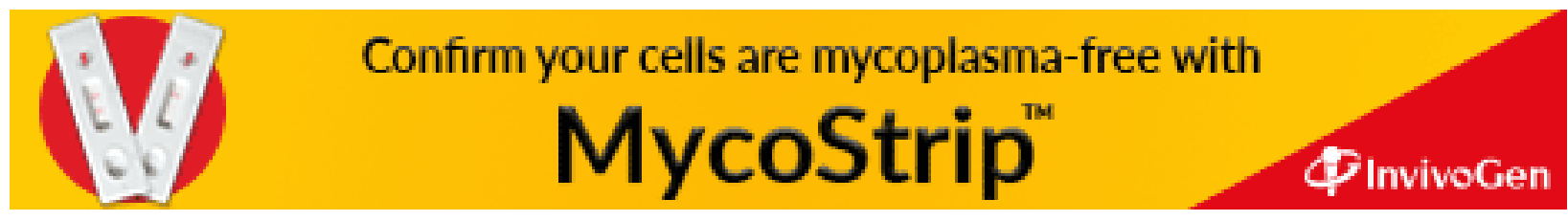

